\title{
Approaches to nonlinear theory creation for machine tools geometric accuracy
}

\author{
Olesya Anikeeva ${ }^{1, *}$, Alexander Ivakhnenko ${ }^{1}$, and Oleg Erenkov ${ }^{2}$ \\ ${ }^{1}$ South-West State University, 305040 Kursk, Russia \\ ${ }^{2}$ Pacific National University, 680035 Khabarovsk, Russia
}

\begin{abstract}
The justification of the nonlinear theory creation in machines geometrical accuracy field is given in the work. The problems accountings of which don't allow providing the consecutive linearization method adequacy at geometrical accuracy calculating of metal-cutting machines are considered. The potential sources of not linearities at calculating of metal-cutting systems accuracy and the sources of the nonlinear members appearance are revealed. At the same time the nonlinear members are considering the machines geometrical errors at a shaping function variation. The full variation of shaping function is defined and the accuracy nonlinear model of the lathe is constructed on the function basis. The directions of further researches in the field of the machines geometrical accuracy nonlinear theory are presented.
\end{abstract}

\section{Introduction}

The variation method for accuracy calculation is widely used [1, 2] at definition of metalcutting machines geometrical accuracy. This method is based on consecutive linearization of shaping function variation and trigonometrical functions from geometrical errors of the knots of the machine form-building system. These errors are small sizes in comparison with the displacement values of the knots of the machine form-building system at details processing. However the situations at which the linearized models don't provide the calculations accuracy have been allocated in work [2]. The situations discussed in the work [2] and other cases when the consecutive linearization method doesn't provide adequacy at calculating of metal-cutting machines geometrical accuracy are considered in this work. The presented situations also known and revealed problems and dependences are the justification to creation of the machines geometrical accuracy nonlinear theory creation. The short state-of-the-art review of the problem and also the solutions proposed by authors of this work are submitted in this work.

\footnotetext{
* Corresponding author: olesya-anikeeva@yandex.ru
} 


\section{Potential sources of not linearities at accuracy calculating of metal-cutting systems}

The two main groups of situations when the linearization method is unsuitable for accuracy calculations have been considered in the work [2] and it is necessary to solve nonlinear problems.

The tasks with significant nonlinear components in the initial function which characterizing the accuracy have been carried to the first group in this work. For example the author considers the admissions IT7-IT9 values of ISO admissions system and the members of trifle second order accounting gives the amendment approximately from 10 to $15 \%$ in comparison with the members only of trifle first order of accounting.

In general this group of situations has been investigated in work [3] at the solution of the admissions synthesis problems for quality indicators of mechanical engineering products and processes and similar estimates (approximately 15\%) had been received.

The admissions synthesis method on values of metal-cutting machines geometrical errors had been created in work [4].The exception of the shaping processes and concrete cutting tools types used at processing when accuracy calculating has been proved for the first time.

In the same work [4] the one linear equation (scalar balance of accuracy) transformation to the three linear equations system for admissions synthesis has been proved. The transformation is realizing on the basis of nonlinear dependence $i=0.45 \sqrt[3]{D}+0.001 D(i-$ admission unit; $D$ - the characteristic size in the range from 1 to $500 \mathrm{~mm}$ ) for various sizes of the treated flat surfaces.

The similar nonlinear problems had been considered in work [5] at admissions multicriteria optimization of machines geometrical accuracy parameters. The Taguti loss function and the production expenses function are used. At the same time the production expenses function considers the manufacturing costs for the machine knots required accuracy achievement.

The second group includes the tasks in which linear components are strictly equal to zero [2]. The solution of these tasks is connected with need of nonlinear members accounting.

The tasks consideration is also executed in work [2] and the next processing errors belong to them:

- Evolvent cogwheels surfaces at their processing by the grinding wheel periphery but not by the grinding wheel end face;

- Hyperbolic surfaces at their turning on the lathe when an axis of workpiece is crossing with the lathe axial line.

In general the situations second group arises at of approximate shaping schemes implementation. These schemes are used for the machine kinematic structure or for the form of the tool cutting edge (surface) simplification, for the elastic deformations sizes decreasing at processing and for the processing productivity increasing, etc.

The common decision of this group tasks had been given in work [6]. The expression for the description of a surface $\boldsymbol{r}^{(\mathbf{2})}$ had been received instead of nominally set surface $\boldsymbol{r}^{(\mathbf{1})}$ at implementation of the of approximate shaping scheme:

$$
r^{(2)}=r^{(1)}+\Delta_{1} k_{1}+\Delta_{2} k_{2}
$$

where $\Delta_{1}$ and $\Delta_{2}$ - the making errors of the shaping scheme; $\boldsymbol{k}_{\mathbf{1}}$ and $\boldsymbol{k}_{\mathbf{2}}-$ single tangent vectors to at the surface $\boldsymbol{r}^{(\mathbf{1})}, \boldsymbol{k}_{1}=\left(\partial \boldsymbol{r}^{(\mathbf{1})} / \partial u\right) /\left|\partial \boldsymbol{r}^{(\mathbf{1})} / \partial u\right|, \boldsymbol{k}_{2}=\left(\partial \boldsymbol{r}^{(\mathbf{1})} / \partial v\right) /\left|\partial \boldsymbol{r}^{(\mathbf{1})} / \partial v\right| ; u, v-$ curvilinear coordinates of the surface of $\boldsymbol{r}^{(\mathbf{1})}$. 
As the errors $\Delta_{1}$ and $\Delta_{2}$ of the shaping scheme in (1) are set rather tangent vectors to a surface of $\boldsymbol{r}^{(\mathbf{1})}$ but not normal to it the sizes of the trifle second order will take part in the surface deviation size $\boldsymbol{r}^{(\mathbf{2})}$ from a surface $\boldsymbol{r}^{(\mathbf{1})}$. If the vectors of $\boldsymbol{k}_{\mathbf{1}}$ and $\boldsymbol{k}_{\mathbf{2}}$ coincide with the respective coordinate lines $u$ or $v$ then the deviations sizes on them don't include in expression (1) since they coincide with the shaping movements.

Thus the first and second situations groups aren't connected with the analysis of nonlinear components inherent to the shaping function variation.

The purpose of this work is consideration of nonlinear components emergence sources at shaping function variation and to identify the problems of their importance assessment.

\section{Sources of not linearities at shaping function variation}

We will consider the first source of nonlinear members emergence at receiving the errors matrix standard form [2]. A solid body change of provision in space is described by an Euler expanded matrix $\boldsymbol{A}_{\boldsymbol{E} \boldsymbol{u}}$ which is function of six parameters $\left(p_{x}, p_{y}, p_{z}, \varphi, \psi, \theta\right)$ :

$$
\boldsymbol{A}_{E u}=\left[\begin{array}{cccc}
\cos \psi \cos \theta & -\cos \varphi \sin \theta & \sin \psi & p_{x} \\
\cos \varphi \sin \theta+\sin \varphi \sin \psi \cos \theta & \cos \varphi \cos \theta-\sin \varphi \sin \psi \sin \theta & -\sin \varphi \cos \psi & p_{y} \\
\sin \varphi \sin \theta-\cos \varphi \sin \psi \cos \theta & \sin \varphi \cos \theta+\cos \varphi \sin \psi \sin \theta & \cos \varphi \cos \psi & p_{z} \\
0 & 0 & 0 & 1
\end{array}\right]
$$

where $p_{x}, p_{y}, p_{z}$ - sizes of a solid body shifts along axes $X, Y$ and $Z$, respectively; $\varphi, \psi, \theta-$ angles of rotation around the same axes.

Replacement of parameters by small sizes of situation and orientation errors in matrix $\boldsymbol{A}_{\boldsymbol{E} \boldsymbol{u}}$, i.e. $\left(p_{x}, p_{y}, p_{z}, \varphi, \psi, \theta\right) \rightarrow(\delta x, \delta y, \delta z, \alpha, \beta, \gamma)$ and trigonometrical expressions linearization concerning them ( $\sin \delta \approx \delta, \cos \delta \approx 1)$ allows to transform expression (2) to $\boldsymbol{W}$ matrix:

$$
\boldsymbol{W}=\boldsymbol{A}_{E u}(\delta x, \delta y, \delta z, \alpha, \beta, \gamma) \approx\left(\begin{array}{cccc}
1 & -\gamma & \beta & \delta x \\
\gamma & 1 & -\alpha & \delta y \\
-\beta & \alpha & 1 & \delta z \\
0 & 0 & 0 & 1
\end{array}\right)
$$

which is represented turn by the sum of two matrixes:

$$
\boldsymbol{W}=\boldsymbol{I}_{\mathbf{4} \times \mathbf{4}}+\boldsymbol{\varepsilon},
$$

where $\boldsymbol{I}_{\mathbf{4} \times \mathbf{4}}-$ a single matrix with dimension $4 \times 4 ; \boldsymbol{\varepsilon}-$ a matrix of errors.

We will notice that the matrix of $\boldsymbol{A}_{\boldsymbol{E} u}$ is the work of six matrixes of elementary movements [1, 2] - $\boldsymbol{A}_{\boldsymbol{E} \boldsymbol{u}}=\boldsymbol{A}^{\mathbf{1}}\left(p_{x}\right) \boldsymbol{A}^{\mathbf{2}}\left(p_{y}\right) \boldsymbol{A}^{\mathbf{3}}\left(p_{z}\right) \boldsymbol{A}^{\mathbf{4}}(\varphi) \boldsymbol{A}^{\mathbf{5}}(\psi) \boldsymbol{A}^{\mathbf{6}}(\theta)$, where $\boldsymbol{A}^{\mathbf{1}}\left(p_{x}\right), \boldsymbol{A}^{\mathbf{2}}\left(p_{y}\right)$ and $\boldsymbol{A}^{3}\left(p_{z}\right)$ - matrixes of a solid body linear shifts along axes $X, Y$ and $Z$, respectively; $\boldsymbol{A}^{4}(\varphi)$, $\boldsymbol{A}^{\mathbf{5}}(\psi)$ and $\boldsymbol{A}^{\mathbf{6}}(\theta)$ - turn matrixes around the same axes.

The order change in the turn matrixes work for example $\boldsymbol{A}=\boldsymbol{A}^{\mathbf{1}}\left(p_{x}\right) \boldsymbol{A}^{\mathbf{2}}\left(p_{y}\right) \boldsymbol{A}^{\mathbf{3}}\left(p_{z}\right) \boldsymbol{A}^{\mathbf{5}}(\psi) \boldsymbol{A}^{\mathbf{6}}(\theta) \boldsymbol{A}^{\mathbf{4}}(\varphi)$ gives other idea instead of expression (2), namely:

$$
\boldsymbol{A}=\left[\begin{array}{cccc}
\cos \psi \cos \theta & -\cos \varphi \cos \psi \sin \theta+\sin \varphi \sin \psi & \sin \varphi \cos \psi \sin \theta+\cos \varphi \sin \psi & p_{x} \\
\sin \theta & \cos \varphi \cos \theta & -\sin \varphi \cos \theta & p_{y} \\
-\sin \psi \cos \theta & \cos \varphi \sin \psi \sin \theta+\sin \varphi \cos \psi & -\sin \varphi \sin \psi \sin \theta+\cos \varphi \cos \psi & p_{z} \\
0 & 0 & 0 & 1
\end{array}\right]
$$


Having performed the matrix $\boldsymbol{A}$ same operations with matrix $\boldsymbol{A}_{\boldsymbol{E} \boldsymbol{u}}$ we will also receive expression (3) for $\boldsymbol{W}$ matrix and that is errors matrix $\boldsymbol{\varepsilon}$ in a linear case will be identical. However if we consider square approach at of trigonometrical functions decomposition ( $\sin \delta \approx \delta, \cos \delta \approx 1-\delta^{2} / 2$ ) then matrixes $\varepsilon$ will be various (tab.).

Table 1. Nonlinear errors matrixes $\varepsilon$.

\begin{tabular}{|c}
\hline \multicolumn{8}{|c|}{ Order of the matrixes work } \\
\hline $\left.\boldsymbol{A}_{\mathbf{1}}=\left[\begin{array}{ccc|cc}-\frac{\beta^{2}+\gamma^{2}}{2} & -\gamma & \beta & \delta x \\
\gamma+\alpha \beta & -\frac{\alpha^{2}+\gamma^{2}}{2} & -\alpha & \delta y \\
\alpha \gamma-\beta & \alpha+\beta \gamma & -\frac{\alpha^{2}+\beta^{2}}{2} & \delta z \\
0 & 0 & 0 & 0\end{array}\right] \mid \boldsymbol{\varepsilon}_{\mathbf{2}}=\left[\begin{array}{cccc}-\frac{\beta^{2}+\gamma^{2}}{2} & -\gamma+\alpha \beta & \beta+\alpha \gamma & \delta x \\
\gamma & -\frac{\alpha^{2}+\gamma^{2}}{2} & -\alpha & \delta y \\
-\beta & \alpha+\beta \gamma & -\frac{\alpha^{2}+\beta^{2}}{2} & \delta z \\
0 & 0 & 0 & 1\end{array}\right]\right)$ \\
\hline
\end{tabular}

We will consider the equation $\boldsymbol{\varepsilon} \boldsymbol{r}=\boldsymbol{\Delta}$ with some set vectors $\boldsymbol{r}=\left(r_{x}, r_{y}, r_{z}, 1\right)^{T}$ and $\Delta=\left(\Delta_{x}, \Delta_{y}, \Delta_{z}, 0\right)^{T}$. It is obvious that various results will be received when using various representations of matrixes $\varepsilon$ containing nonlinear members. There are in total six shifts at calculation of the work $\boldsymbol{A}^{\mathbf{4}}(\varphi), \boldsymbol{A}^{\mathbf{5}}(\psi)$ и $\boldsymbol{A}^{\mathbf{6}}(\theta)$ and it is impossible to recognize reasonable use of Euler expanded matrix $\boldsymbol{A}_{\boldsymbol{E} \boldsymbol{u}}$ when determining errors matrix with nonlinear components. The direct problem solution of the accuracy calculation i.e. finding of the vector $\Delta$ components at known values of elementary errors $\delta x, \delta y, \delta z, \alpha, \beta$ and $\gamma$ at any representation of the matrix $\boldsymbol{\varepsilon}$ for the considered equation is easy. The return problem of accuracy calculation can has set of decisions that will present difficulties as the number of unknown are more than quantity of the equations and approximate or numerical methods will be used.

We will consider the second source of nonlinear members emergence in machines accuracy calculations. For the set shaping function $\boldsymbol{r}_{\mathbf{0}}=\prod_{i=1}^{n} \boldsymbol{A}_{\boldsymbol{i}} \boldsymbol{r}_{\boldsymbol{p} u}$, where $\boldsymbol{A}_{\boldsymbol{i}}$ - matrixes of shifts and turns; $\boldsymbol{r}_{\boldsymbol{p}}$ - the radius vector of the tool cutting edge; $n$ - the number of knots of the machine form-building system and including the cutting tool ideal we will define its variation:

$$
\Delta \boldsymbol{r}_{\mathbf{0}}=\left(\boldsymbol{\varepsilon}_{\mathbf{0}} \prod_{i=1}^{n} \boldsymbol{A}_{i}\left(\boldsymbol{I}+\boldsymbol{\varepsilon}_{\boldsymbol{i}}\right)+\prod_{i=1}^{n} \boldsymbol{A}_{\boldsymbol{i}}\left(\boldsymbol{I}+\boldsymbol{\varepsilon}_{\boldsymbol{i}}\right)-\prod_{i=1}^{n} \boldsymbol{A}_{\boldsymbol{i}}\right) \boldsymbol{r}_{p u} .
$$

Unlike work [2] we won't carry out linearization of the matrixes work i.e. to consider that $\boldsymbol{A}_{\boldsymbol{i}} \boldsymbol{\varepsilon}_{\boldsymbol{i}} \boldsymbol{A}_{\boldsymbol{j}} \boldsymbol{\varepsilon}_{\boldsymbol{j}} \approx \mathbf{0}$, where $\mathbf{0}$ - a zero matrix. We will note that expression (6) structurally corresponds to the representation of the shaping function variation entered in work [7] in form $\Delta r_{0}=\varepsilon_{0} r_{0}+d r_{0}+\delta r_{0}$.

Thus the two sources of the nonlinear members appearance considering the machines geometrical errors at the shaping function variation are revealed and the first source is parametrical and the second is structural. 


\section{Nonlinear model of the lathe accuracy}

The lathe shaping function with the dot cutting tool is equal to $\boldsymbol{r}_{\mathbf{0}}=\boldsymbol{A}^{\mathbf{6}}(\varphi) \boldsymbol{A}^{\mathbf{3}}(z) \boldsymbol{A}^{\mathbf{1}}(x) \boldsymbol{e}^{\mathbf{4}}$ where $e^{4}=(0 ; 0 ; 0 ; 1)^{T}$. The variation of this function with accuracy to square members has the appearance:

$$
\begin{gathered}
\Delta r_{0} \approx\left(\boldsymbol{\varepsilon}_{0} \boldsymbol{A}^{\mathbf{6}}(\varphi) \boldsymbol{A}^{\mathbf{3}}(z) \boldsymbol{A}^{\mathbf{1}}(x)+\boldsymbol{A}^{\mathbf{6}}(\varphi) \boldsymbol{\varepsilon}_{1} \boldsymbol{A}^{\mathbf{3}}(z) \boldsymbol{A}^{\mathbf{1}}(x)+\boldsymbol{A}^{\mathbf{6}}(\varphi) \boldsymbol{A}^{\mathbf{3}}(z) \boldsymbol{\varepsilon}_{2} \boldsymbol{A}^{\mathbf{1}}(x)+\right. \\
+\boldsymbol{A}^{\mathbf{6}}(\varphi) \boldsymbol{A}^{\mathbf{3}}(z) \boldsymbol{A}^{\mathbf{1}}(x) \boldsymbol{\varepsilon}_{3}+\boldsymbol{\varepsilon}_{0} \boldsymbol{A}^{\mathbf{6}}(\varphi) \boldsymbol{\varepsilon}_{1} \boldsymbol{A}^{\mathbf{3}}(z) \boldsymbol{A}^{\mathbf{1}}(x)+\boldsymbol{\varepsilon}_{0} \boldsymbol{A}^{\mathbf{6}}(\varphi) \boldsymbol{A}^{\mathbf{3}}(z) \boldsymbol{\varepsilon}_{2} \boldsymbol{A}^{\mathbf{1}}(x)+ \\
+\boldsymbol{\varepsilon}_{\mathbf{0}} \boldsymbol{A}^{\mathbf{6}}(\varphi) \boldsymbol{A}^{\mathbf{3}}(z) \boldsymbol{A}^{\mathbf{1}}(x) \boldsymbol{\varepsilon}_{3}+\boldsymbol{A}^{\mathbf{6}}(\varphi) \boldsymbol{\varepsilon}_{\mathbf{1}} \boldsymbol{A}^{\mathbf{3}}(z) \boldsymbol{\varepsilon}_{2} \boldsymbol{A}^{\mathbf{1}}(x)+ \\
\left.+\boldsymbol{A}^{\mathbf{6}}(\varphi) \boldsymbol{\varepsilon}_{\mathbf{1}} \boldsymbol{A}^{\mathbf{3}}(z) \boldsymbol{A}^{\mathbf{1}}(x) \boldsymbol{\varepsilon}_{3}+\boldsymbol{A}^{\mathbf{6}}(\varphi) \boldsymbol{A}^{\mathbf{3}}(z) \boldsymbol{\varepsilon}_{\mathbf{2}} \boldsymbol{A}^{\mathbf{1}}(x) \boldsymbol{\varepsilon}_{3}\right) \boldsymbol{r}_{\boldsymbol{p}} .
\end{gathered}
$$

The first four members in expression (7) correspond to the linearized representation of the shaping function variation and other six square members consider mutual works of the machine geometrical errors. The influence of separate geometrical errors at the linearized representation $\Delta \boldsymbol{r}_{0}$ for the lathe is rather well investigated [1,2] unlike influence of square members on the size, form and displacement errors at various surfaces processing.

\section{The further researches directions in the field of the machines geometrical accuracy nonlinear theory}

The first direction of further researches consists in necessity to solve the problem of initial matrix choice justification. The initial matrix transforms the solid body movement and is the basis for geometrical errors matrix development at accounting of parametrical not linearities and also for identification of the square members importance at the solution of the accuracy calculations return problems.

The research of the influence and the structural not linearities importance in the shaping function variation on formation of the size, form and displacement errors at various surfaces processing are the second direction of further researches.

\section{Conclusions}

The justification of the nonlinear theory creation in machines geometrical accuracy field is given in the work. The problems accountings of which don't allow providing the consecutive linearization method adequacy at geometrical accuracy calculating of metalcutting machines are considered. The parametrical and structural sources of the nonlinear members appearance are revealed and these members considering the machines geometrical errors at the shaping function variation. The nonlinear model of the lathe accuracy is developed. The two directions of further researches in the field of the machines geometrical accuracy nonlinear theory are defined.

The work is executed with financial support of RFBR (the Project № 16-38-60049).

\section{References}

1. D.N. Reshetov, V.T. Portman, Accuracy of Machine Tools (ASME Press, NY, 1988)

2. V.T. Portman, Mechanics of accuracy in engineering design of machines and robots. Volume I: Nominal functioning and geometric accuracy (ASME Press, NY, 2018)

3. A.A. Ivakhnenko, The synthesis of tolerances indicators of quality in the design and operation of engineering products (The dissertation on competition of candidate of technical Sciences, SWSU, 2015) 
4. O.V. Anikeeva, The synthesis of tolerances of the machine tools geometric accuracy parameters, Bulletin of Voronezh state technical University, v. 13, № 6, pp. 95-103 (2017)

5. Hong Yihai, Liu Jiangnan, Yang Xiaojuan, Jiang Guang, Analysis and Robust Design of Geometric Accuracy of a Three-Axis CNC Surface Grinding Machine, The 14th IFToMM World Congress, Taipei, Taiwan (2015) DOI Number: 10.6567/IFToMM.14TH.WC.PS12.001

6. A.G. Ivakhnenko, Conceptual design of metal-cutting systems. Structural synthesis: monograpf (Habarovsk, 1998)

7. O.V. Anikeeva, A hierarchical system of functional relationships in the cutting machines, Fundamental and Applied Problems of Engineering and Technology, v. 6 (326), pp. 42-51 (2017) 